\title{
Erratum to: Non-hyperelliptic Riemann surfaces with real field of moduli but not definable over the reals
}

\author{
RubÉn A. HidAlgo
}

Mathematics Subject Classification. Primary 30F10, 30F40.

Keywords. Riemann surfaces, Algebraic curves, Automorphisms, Fields of moduli.

Erratum to: Arch. Math. 93 (2009), 219-224

DOI 10.1007/s00013-009-0025-4

In the original article the following theorem was stated (with $\lambda_{1}=\lambda$ and $\lambda_{2}=\mu$.).

Theorem 1. Let $\lambda \in \mathbb{R}$ and $\mu \in \mathbb{C}$ so that $\lambda<-(3+2 \sqrt{2}), \operatorname{Im}(\mu)>0, \operatorname{Re}(\mu)<$ 0 , and $|\mu|^{2}=-\lambda$; and consider the non-singular projective algebraic curve

$$
C_{\lambda, \mu}=\left\{\begin{array}{r}
x_{1}^{2}+x_{2}^{2}+x_{3}^{2}=0 \\
\lambda x_{1}^{2}+x_{2}^{2}+x_{4}^{2}=0 \\
\mu x_{1}^{2}+x_{2}^{2}+x_{5}^{2}=0 \\
-\mu x_{1}^{2}+x_{2}^{2}+x_{6}^{2}=0
\end{array}\right\} \subset \mathbb{P}^{5}
$$

The Riemann surface $C_{\lambda, \mu}$ is a non-hyperelliptic Riemann surface of genus 17, which admits an anticonformal automorphism of order 4, but does not admit an anticonformal involution. In particular, $C_{\lambda, \mu}$ has its field of moduli contained in $\mathbb{R}$ but none of its fields of definition are contained in $\mathbb{R}$. 
Unfortunately, as stated, inside the two-dimensional real family of curves as described in the previous theorem there may be a one-dimensional real locus of such curves which do not satisfy the ensured properties. The problem is that in Section 3.2.2. in original article we assumed that there are only two subsets of cardinality four of $\{\infty, 0,1, \lambda, \mu,-\mu\}$ each one being a subset of some circle. This is not true and there are three of such subsets. We provide the correct arguments that replace (the first half of) Section 3.2.2. The correct statement of the theorem should be the following.

Theorem 2. Let $\theta$ be so that $e^{i \theta} \notin\{ \pm 1, \pm i\}$. There is exactly one point $\lambda_{\theta} \in$ $(-\infty,-1)$ so that, if $\lambda \in(-\infty,-1)-\left\{\lambda_{\theta}\right\}$ and $\mu=\sqrt{|\lambda|} e^{i \theta}$, then $C_{\lambda, \mu}$ is a non-hyperelliptic Riemann surface of genus 17 which admits an anticonformal automorphism of order 4, but does not admit an anticonformal involution. In particular, $C_{\lambda, \mu}$ has its field of moduli contained in $\mathbb{R}$ but none of its fields of definition are contained in $\mathbb{R}$.

In order to clarify the existence of the unique value $\lambda_{\theta}$, we need to recall some basic facts.

A generalized circle in the Riemann sphere $\widehat{\mathbb{C}}$ is either an Euclidian circle in $\mathbb{C}$ or the union of $\infty$ with an Euclidian line in $\mathbb{C}$. Given four different points $a, b, c, d \in \widehat{\mathbb{C}}$, it is defined the cross-ratio $[a, b, c, d]=T(d)$, where $T$ is a the unique Möbius transformation satisfying that $T(a)=$ $\infty, T(b)=0$ and $T(c)=1$. By the definition, $[a, b, c, d] \in \mathbb{C}-\{0,1\}$. If $S$ is any Möbius transformation, then $[S(a), S(b), S(c), S(d)]=[a, b, c, d]$. The points $a, b, c, d$ belong to a common generalized circle if and only if $[a, b, c, d] \in \mathbb{R}$. In particular, Möbius transformations send generalized circles into generalized circles. Any permutation of the four points changes the value of $[a, b, c, d]$ to a value $R([a, b, c, d])$, were $R \in \mathbb{G}=\langle A(z)=$ $1 / z, B(z)=z /(z-1)\rangle \cong \mathfrak{S}_{3}$. In particular, if $[a, b, c, d] \in\{-1,1 / 2,2\}$, then the cross-ratio of any permutation of these four points still in the same set. If $a \neq 0, \infty$, then $[\infty, 0, a,-a]=-1$. The only cross-ratios, obtained by permutation of $\infty, 0, a$ and $-a$, producing the same value of -1 are given by $[\infty, 0, a,-a],[\infty, 0,-a, a],[0, \infty, a,-a],[0, \infty,-a, a],[a,-a, \infty, 0]$, $[-a, a, \infty, 0],[a,-a, 0, \infty]$ and $[-a, a, 0, \infty]$.

The first half of Section 3.2.2. in the original article should be replaced by the following lines.

Let us assume that $e^{i \theta} \notin\{ \pm 1\}, \lambda=-r^{2} \in \mathbb{R}$ with $r>1$ and $\mu=r e^{i \theta}$. By direct inspection at the cross-ratios, with the above restrictions on the parameters $\lambda$ and $\mu$, we may notice that the only subsets of cardinality 4 of $\{\infty, 0,1, \lambda, \mu,-\mu\}$ contained in a generalized circle are given by

$$
\{\infty, 0,1, \lambda\}, \quad\{\infty, 0, \mu,-\mu\}, \quad\{1, \lambda, \mu,-\mu\} .
$$

The respective cross-ratios are given by

$$
\begin{gathered}
{[\infty, 0,1, \lambda]=\lambda \notin\{-1,1 / 2,2\}} \\
{[\infty, 0, \mu,-\mu]=-1}
\end{gathered}
$$




$$
[1, \lambda, \mu,-\mu]=-\frac{\left.r^{4}+2\left(2 \sin (\theta)^{2}-1\right) r^{2}+1\right)}{\left(r^{2}+2 \cos (\theta) r+1\right)^{2}} \notin\{-1,1 / 2,2\}
$$

Let $\theta$ and $R \in \mathbb{G}$ be fixed. The equation $[1, \lambda, \mu,-\mu]=R(\lambda)$ is equivalent to a polynomial equation $P_{\theta, R}(r)=0$, where $P_{\theta, R}(x) \in \mathbb{R}[x]$ is a non-constant real polynomial of degree either 2 or 4 . These polynomials $P_{\theta, R}(x)$ are given by the following ones:

$$
\begin{aligned}
& x^{2}+2 \cos (\theta) x-1 ; x^{2}-2 \cos (\theta) x-1 ; 2 x^{4}+3 x^{2}-2 \cos (\theta) x+1 ; \\
& 2 x^{4}+3 x^{2}+2 \cos (\theta) x+1 ; x^{4}+2 \cos (\theta) x^{3}+3 x^{2}+2 ; x^{4}-2 \cos (\theta) x^{3} \\
& \quad+3 x^{2}+2
\end{aligned}
$$

The degree four polynomials have no real zeroes greater than 1 . The two degree two polynomials have real zeroes greater than 1 , respectively, at the points $r_{1}(\theta)=\sqrt{1+\cos (\theta)^{2}}-\cos (\theta)$ and $r_{2}(\theta)=\sqrt{1+\cos (\theta)^{2}}+\cos (\theta)$. Notice that $r_{1}(\theta) r_{2}(\theta)=1$, and that $r_{1}(\pi / 2+k \pi)=r_{2}(\pi / 2+k \pi)=1$, for each integer $k$. In this way, we obtain that for each value of $\theta$, with $e^{i \theta} \notin\{ \pm 1, \pm i\}$ the set

$$
Q_{\theta}=\left\{r_{1}(\theta), r_{2}(\theta)\right\} \cap(1, \infty)
$$

has cardinality 1 ; say $Q_{\theta}=\left\{r_{\theta}\right\}$. For instance, $Q_{\pi / 3}=\{(1+\sqrt{5}) / 2\}$.

So, if we set $\lambda_{\theta}=-r_{\theta}^{2}$, then for $\lambda \in(-\infty,-1)-\left\{\lambda_{\theta}\right\}$, the cross-ratios of the three collections of points as above are non-equivalent under the action of $\mathbb{G}$. In particular, if $T$ is a Möbius transformation so that

$$
\{\infty, 0,1, \lambda, \mu,-\mu\} \stackrel{T}{\mapsto}\{\infty, 0,1, \lambda, \mu,-\mu\},
$$

then

$$
\{\infty, 0,1, \lambda\} \stackrel{T}{\mapsto}\{\infty, 0,1, \lambda\}, \quad\{\infty, 0, \mu,-\mu\} \stackrel{T}{\mapsto}\{\infty, 0, \mu,-\mu\} .
$$

Now, the above together with the last paragraph of Section 3.2.2. in the original article provides the correct proof.

\section{Rubén A. Hidalgo}

Departamento de Matemáticas, Universidad Técnica Federico Santa María, Valparaíso, Chile e-mail: ruben.hidalgo@usm.cl 